RAIRO-Oper. Res.55 (2021) S699-S704

RAIRO Operations Research

https://doi.org/10.1051/ro/2020004

www.rairo-ro.org

\title{
THE SUPER-CONNECTIVITY OF ODD GRAPHS AND OF THEIR KRONECKER DOUBLE COVER
}

\author{
Gülnaz Boruzanli Ekinci ${ }^{1}$ And John Baptist Gauci ${ }^{2, *}$
}

\begin{abstract}
The study of connectivity parameters forms an integral part of the research conducted in establishing the fault tolerance of networks. A number of variations on the classical notion of connectivity have been proposed and studied. In particular, the super-connectivity asks for the minimum number of vertices that need to be deleted from a graph in order to disconnect the graph without creating isolated vertices. In this work, we determine this value for two closely related families of graphs which are considered as good models for networks, namely the odd graphs and their Kronecker double cover. The odd graphs are constructed by taking all possible subsets of size $k$ from the set of integers $\{1, \ldots, 2 k+1\}$ as vertices, and defining two vertices to be adjacent if the corresponding $k$-subsets are disjoint; these correspond to the Kneser graphs $K G(2 k+1, k)$. The Kronecker double cover of a graph $G$ is formed by taking the Kronecker product of $G$ with the complete graph on two vertices; in the case when $G$ is $K G(2 k+1, k)$, the Kronecker double cover is the bipartite Kneser graph $H(2 k+1, k)$. We show that in both instances, the super-connectivity is equal to $2 k$.
\end{abstract}

Mathematics Subject Classification. 05C40, 94C15, 05D05.

Received March 11, 2019. Accepted January 3, 2020.

\section{INTRODUCTION}

The family of odd graphs was introduced in 1917 by Kowaleski [17], although the name was only coined much later. One generalisation of the concept underlying odd graphs is given by the family of Kneser graphs, introduced by Lovász in his proof of Kneser's conjecture [18]. For any two integers $k \geq 1$ and $n \geq 2 k+1$, the Kneser graph $K G(n, k)$ has the $k$-element subsets of $[n]:=\{1,2, \ldots, n\}$ as vertices and any pair of disjoint subsets forms an edge. Thus, $K G(n, k)$ has $\left(\begin{array}{l}n \\ k\end{array}\right)$ vertices and is regular of degree $\left(\begin{array}{c}n-k \\ k\end{array}\right)$. It is well-known that if $n<2 k$, then $K G(n, k)$ is a null graph, while if $n=2 k$, then $K G(n, k)$ consists of $k$ disjoint copies of the complete graph on two vertices. The Kneser graph $K G(n, 1)$ is isomorphic to the complete graph on $n$ vertices, and hence, when referring to Kneser graphs, we shall always assume that $k \geq 2$.

The Kronecker double cover (or, equivalently, the bipartite double cover) of the Kneser graph $K G(n, k)$ is known as the bipartite Kneser graph $H(n, k)$. More formally, for any two integers $k \geq 1$ and $n \geq 2 k+1$, the bipartite Kneser graph $H(n, k)$ has all the $k$-element subsets and all the $(n-k)$-element subsets of $[n]$ as vertices, and two vertices are adjacent if and only if one of them is a subset of the other. It follows that $H(n, k)$ has $2\left(\begin{array}{c}n \\ k\end{array}\right)$

Keywords. Connectivity, super-connectivity, odd graph, Kneser graph, bipartite Kneser graph, Kronecker double cover.

1 Department of Mathematics, Ege University, Bornova, 35100, Izmir, Turkey.

2 Department of Mathematics, Faculty of Science, University of Malta, Msida, Malta.

* Corresponding author: john-baptist.gauci@um.edu.mt 
vertices and is regular of degree $\left(\begin{array}{c}n-k \\ k\end{array}\right)=\left(\begin{array}{c}n-k \\ n-2 k\end{array}\right)$. The reason why we chose $n \geq 2 k+1$ is that $H(2 k, k)$ is, in fact, a null graph.

It has long been conjectured that the Kneser graphs and the bipartite Kneser graphs have a Hamiltonian cycle, apart from one notorious exception, namely the Petersen graph $K G(5,2)$. The sparsest among these graphs (that is, those graphs for which the ratio of the number of edges to the number of vertices is smallest) are respectively $K G(2 k+1, k)$ and $H(2 k+1, k)$. In the myriad of papers on the subject, these two families have received particular attention because proving Hamiltonicity for the sparsest cases is particularly intricate [22].

In this work we focus our attention on these two families of graphs, that is, the Kneser graph $K G(2 k+1, k)$ and the bipartite Kneser graph $H(2 k+1, k)$. The former is the odd graph $O_{k+1}$, where, for any integer $k \geq 2$, the vertex-set corresponds to the set of all possible $k$-element subsets of a $(2 k+1)$-element set, and two vertices are adjacent if and only if the corresponding $k$-subsets are disjoint. The latter is sometimes referred to as the revolving door graph, the middle-levels graph [11], the middle cube $M Q_{2 k+1}=Q_{2 k+1}(k, k+1)$ [10], or the regular hyperstar graph $H S(2(k+1), k+1)$ [16,20,21].

A graph $G$ is vertex-transitive (resp. edge-transitive) when, for every pair of vertices $u, v \in V(G)$ (resp. edges $e_{1}, e_{2} \in E(G)$ ), there is an automorphism that maps $u$ to $v$ (resp. $e_{1}$ to $e_{2}$ ). If a graph $G$ is both vertexand edge-transitive, then $G$ is symmetric. It is usually desirable that interconnection networks are modelled using symmetric graphs [15], because vertex-transitivity permits the implementation of the same routing and communication schemes at each vertex (or node) of the network, whereas edge-transitivity allows recursive constructions to be used. In 1987, Chen and Lih showed that the Kneser graphs are symmetric [9], while the bipartite Kneser graphs were shown to be symmetric by Mirafzal and Zafari [21].

Apart from symmetry, another property that is usually desirable when modelling interconnection networks is regularity as this simplifies their study in terms of diameter and diameter vulnerability problems. Many large interconnection networks are modelled by sparse graphs so as to keep the "expense" of linking the nodes to a minimum. Social, biological, computer and transportation networks are only a few examples of such real networks. However, connectivity parameters are also generally taken into consideration when modelling these networks as high connectivity makes them more tolerant to faults.

Ensuring and preserving connectivity is, in fact, vital in most networks. It is thus fully apprehensible that connectivity studies are a fundamental topic that still attracts a great deal of consideration in the field of combinatorial optimisation. The property of a graph being highly connected depends solely on the number of paths that the graph contains between every pair of vertices. Besides the classical connectivity measures that study the minimum number of vertices or edges that need to be deleted to disconnect the graph, other types of connectivity have recently received much attention. These include connectivity parameters that impose some restrictions on the components of the remaining graph; a notion proposed by Harary [14] and known as conditional connectivity.

In particular, in this work we restrict our attention to the super-connectivity of a graph as it is argued by many (see e.g. [2]) that this parameter provides a better measure of the reliability of a network. This notion studies the least number of vertices that need to be deleted from a graph to disconnect the graph such that each remaining component is not trivial, that is, each component is restricted to contain at least two adjacent vertices. More formally, the super-connectivity of a graph $G$ is the size of a minimum vertex-cut $S$ (that is, a vertex-cut of smallest cardinality over all vertex-cuts of $G$ ) such that $G-S$ is disconnected and has no isolated vertices. If such a vertex-cut exists, it is referred to as a super vertex-cut and its cardinality is denoted by $\kappa^{\prime}=\kappa^{\prime}(G)$; otherwise we write $\kappa^{\prime}(G)=+\infty$. A graph $G$ is super-connected if every minimum vertex-cut is made up of the neighbourhood $N_{G}(x)$ of a vertex $x \in V(G)$, where $N_{G}(x)=\{y \in V(G): x y \in E(G)\}$. In this case, $\kappa^{\prime}$ is strictly greater than the connectivity $\kappa=\kappa(G)$ of $G$; otherwise $\kappa^{\prime}=\kappa$. Some examples of graph classes which have been analysed for their super-connectivity are circulant graphs [3], products of various graphs (see $[7,8,12,19]$, and the references therein), hypercubes [13,24,25], generalized Petersen graphs [4], Johnson graphs [5] and Kneser graphs [1,6].

In the sequel, we consider the odd graphs and their Kronecker double cover. In Section 2, we present some preliminary results pertaining to our work on super-connectivity. This parameter is then determined for the 
sparsest Kneser graphs in Section 3, thereby proving part of the conjecture in [6], namely that $\kappa^{\prime}(K G(2 k+$ $1, k))=2 k$ for $k \geq 2$. In order to facilitate a direct comparison with the aforementioned conjecture, when discussing odd graphs we shall thus use the notation pertaining to Kneser graphs. In Section 4, we shift our attention to the sparsest bipartite Kneser graphs and determine their super-connectivity.

\section{Preliminaries}

Two fundamental properties of a minimum super vertex-cut of a connected graph $G$ are presented in the following remark. Whereas the first property follows immediately since otherwise $G$ would be disconnected, the second one revolves around the minimality of the super vertex-cut.

Remark 2.1. A minimum super vertex-cut $S$ of $G$ contains a vertex having at least a neighbour in every component of $G-S$. Moreover, if a vertex $v$ in a minimum super vertex-cut $S$ of $G$ has a neighbour in one component of $G-S$, then it has at least one neighbour in every component of $G-S$.

Watkins proved that if a connected simple graph $G$ is edge-transitive and regular, then the connectivity of $G$ is maximum [23]. Thus, the connectivity of Kneser graphs and of bipartite Kneser graphs follow immediately.

Theorem 2.2. The connectivity of the Kneser graph $K G(n, k)$ for $n>2 k$ and $k \geq 1$ is $\left(\begin{array}{c}n-k \\ k\end{array}\right)$.

Theorem 2.3. The connectivity of the bipartite Kneser graph $H(n, k)$ for $n \geq 2 k$ and $k \geq 1$ is $\left(\begin{array}{c}n-k \\ k\end{array}\right)$.

To simplify the notation used, we adopt the same convention introduced in [5]. A vertex $x$ corresponding to the $r$-subset $\{1, \ldots, r\} \in\left(\begin{array}{c}{[n]} \\ r\end{array}\right)$ will be denoted by $x=z_{1} \ldots z_{r}$, where $z_{1}, \ldots, z_{r}$ are referred to as the entries of $x$. We shall denote by $x_{i}^{j}$ the vertex obtained from the vertex $x$ by removing the entry $z_{i}$ from $x$ and introducing a new entry $z_{j}$ which is not in $x$. For instance, if $x=z_{1} \ldots z_{r}$, then $x_{1}^{r+1}=\{1, \ldots, r\} \backslash\{1\} \cup\{r+1\}=z_{2} \ldots z_{r+1}$. This process can be repeated in such a way that $x_{i, h}^{j, \ell}=\left(x_{i}^{j}\right)_{h}^{\ell}$, where the entry $z_{h}$ is removed from $x_{i}^{j}$ and the entry $z_{\ell}$ is introduced in $x_{i}^{j}$.

\section{The SUPER-CONNECTIVITY OF ODD GRAPHS}

In Theorem 3.1, we first establish a lower bound for $\kappa^{\prime}(K G(2 k+1, k))$ and then show that it is possible to find a super vertex-cut that attains this value.

Theorem 3.1. The super-connectivity of the odd graph $K G(2 k+1, k)$ is $2 k$, where $k \geq 2$.

Proof. The odd graph $K G(5,2)$ is the well-known Petersen graph, which has super-connectivity equal to 4 [4]. Thus, we let $k \geq 3$.

Let $S$ be a minimum super vertex-cut of $\mathcal{G}=K G(2 k+1, k)$ and let $C_{1}$ and $C_{2}$ be two components of $\mathcal{G}-S$. Let $x$ be a vertex of $S$, say $x=z_{1} \ldots z_{k}$. By Remark 2.1, $x$ is adjacent to at least a vertex $w$ in $C_{1}$ and a vertex $y$ in $C_{2}$. Without loss of generality, we let $w=z_{k+1} \ldots z_{2 k}$ and $y=z_{k+1} \ldots z_{2 k-1} z_{2 k+1}=w_{2 k}^{2 k+1}$.

Each component of $\mathcal{G}-S$ contains at least two adjacent vertices. We let $v$ be the vertex adjacent to $w$ in $C_{1}$ and $u$ be the vertex adjacent to $y$ in $C_{2}$. Since $v$ is a neighbour of $w$ which is different from $x$, then $z_{2 k+1}$ is one of the entries of $v$ and the remaining $k-1$ entries of $v$ are to be chosen from the set $\left\{z_{1}, \ldots, z_{k}\right\}$. Without loss of generality, let $v=z_{2} \ldots z_{k} z_{2 k+1}=x_{1}^{2 k+1}$. Similarly, we note that $z_{2 k}$ must be one of the entries of $u$ and that the remaining $k-1$ entries of $u$ are to be chosen from the set $\left\{z_{1}, \ldots, z_{k}\right\}$. Hence, there are two separate cases to consider, depending on whether $|u \cap v|=k-1$ or $|u \cap v|=k-2$. In the sequel, we consider each case separately and construct $2 k$ internally vertex-disjoint paths between the vertices of $C_{1}$ and the vertices of $C_{2}$, thus showing that $|S| \geq 2 k$. 
Case 1. $|u \cap v|=k-1$, in which case $u=x_{1}^{2 k}$. Consider the following paths:

(1.1) $w \sim x \sim y$

(1.2) $w \sim x_{i}^{2 k+1} \sim w_{2 k}^{i} \sim x_{i}^{2 k} \sim y$, for all $i \in\{2, \ldots, k\}$

(1.3) $v \sim w_{2 k}^{1} \sim u$

(1.4) $v \sim w_{j}^{1} \sim x_{1}^{j} \sim w_{j, 2 k}^{1,2 k+1} \sim u$, for all $j \in\{k+1, \ldots, 2 k-1\}$.

Case 2. $|u \cap v|=k-2$, in which case $u=x_{i}^{2 k}$ for any $i \in\{2, \ldots, k\}$, say $u=x_{2}^{2 k}$. Consider the following paths:

(2.1) $w \sim x \sim y$

(2.2) $w \sim x_{i}^{2 k+1} \sim w_{2 k}^{i} \sim x_{i}^{2 k} \sim y$, for all $i \in\{3, \ldots, k\}$

(2.3) $v \sim w_{2 k}^{1} \sim x_{1}^{2 k} \sim y$

(2.4) $w \sim x_{2}^{2 k+1} \sim w_{2 k}^{2} \sim u$

(2.5) $v \sim w_{j}^{1} \sim x_{1}^{j} \sim w_{j, 2 k}^{1,2 k+1} \sim x_{1,2}^{j, 2 k} \sim w_{j, 2 k}^{2,2 k+1} \sim u$, for all $j \in\{k+1, \ldots, 2 k-1\}$.

In each of the above two cases, it can be readily checked that there are $2 k$ paths and they are all internally disjoint. Thus, $\kappa^{\prime}(\mathcal{G}) \geq 2 k$.

Since $\mathcal{G}$ is regular of degree $k+1$ and no two adjacent vertices share a common neighbour, then we can take the neighbours of any two adjacent vertices $a$ and $b$ to form a super vertex-cut $S$. In order to see that $\mathcal{G}-S$ does not contain isolated vertices, suppose, for contradiction, that $c$ is an isolated vertex in $\mathcal{G}-S$. This implies that all its $k+1$ neighbours are in $S$ and, thus, it has at least one neighbour which is a neighbour of $a$ and at least another neighbour which is a neighbour of $b$. Thus $\mathcal{G}$ has a cycle of length 5 . However, the girth of the odd graph $K G(2 k+1, k)$ is 6 for $k \geq 3$, a contradiction. Thus $\kappa^{\prime}(\mathcal{G}) \leq|S|=2 k$ and the result follows.

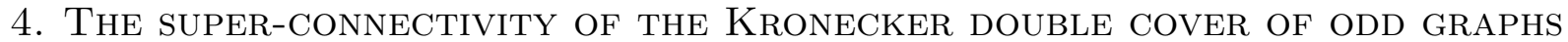

Since $H(n, k)$ is a bipartite graph, we let $V(H(n, k))=V_{1} \cup V_{2}$ such that $V_{1}=\{v \in V(H(n, k)):|v|=k\}$ and $V_{2}=\{v \in V(H(n, k)):|v|=n-k\}$.

Let $S$ be a minimum super vertex-cut of $\mathcal{G}=H(2 k+1, k)$, where $k \geq 2$. By Remark 2.1, there exists a vertex $x \in S$ having a neighbour in each component of $\mathcal{G}-S$. Since the graph $\mathcal{G}$ is bipartite, there are two cases to consider: either $x$ is in $V_{1}$ (Lem. 4.1) or $x$ is in $V_{2}$ (Lem. 4.2).

Lemma 4.1. Let $S$ be a minimum super vertex-cut of $\mathcal{G}=H(2 k+1, k)$, where $k \geq 2$. If $S$ contains a vertex $x$ such that $x$ has a neighbour in every component of $\mathcal{G}-S$ and $x \in V_{1}$, then $|S| \geq 2 k$.

Proof. Since $x \in V_{1}$, then $|x|=k$, say $x=z_{1} \ldots z_{k}$. Let a neighbour of $x$ in a component $C_{1}$ be $w$ and let a neighbour of $x$ in a component $C_{2}$ be $y$. By definition, $w$ and $y$ are in $V_{2}$, and $x$ is contained in both $w$ and $y$. Thus, without loss of generality, let $w=z_{1} \ldots z_{k+1}$ and $y=z_{1} \ldots z_{k} z_{k+2}=w_{k+1}^{k+2}$.

Since $S$ is a super vertex-cut, the components $C_{1}$ and $C_{2}$ are non-trivial. Thus, there is a vertex $v$ adjacent to $w$ in $C_{1}$ such that $v \in V_{1}$ and, similarly, there is a vertex $u$ adjacent to $y$ in $C_{2}$ such that $u \in V_{1}$. We remark that $v \subset w$ and $u \subset y$. Since the vertices $u$ and $v$ are different from $x$, then $v$ and $u$ contain the entries $z_{k+1}$ and $z_{k+2}$, respectively, and $\left|u \cap\left\{z_{1}, \ldots, z_{k}\right\}\right|=\left|v \cap\left\{z_{1}, \ldots, z_{k}\right\}\right|=k-1$. Without loss of generality, we let $v=z_{1} \ldots z_{k-1} z_{k+1}=x_{k}^{k+1}$. This implies that there are two separate cases to consider, depending on whether $|u \cap v|=k-1$ or $|u \cap v|=k-2$.

Case 1. $|u \cap v|=k-1$, in which case $u=x_{k}^{k+2}$. Consider the following paths:

(1.1) $w \sim x \sim y$

(1.2) $w \sim x_{i}^{k+1} \sim w_{i}^{k+2} \sim x_{i}^{k+2} \sim y$, for all $i \in\{1, \ldots, k-1\}$

(1.3) $v \sim w_{k}^{k+2} \sim u$

(1.4) $v \sim w_{k}^{j} \sim x_{k}^{j} \sim w_{k, k+1}^{j, k+2} \sim u$, for all $j \in\{k+3, \ldots, 2 k+1\}$. 
Case 2. $|u \cap v|=k-2$, in which case $u=x_{i}^{k+2}$ for any $i \in\{1, \ldots, k-1\}$. Without loss of generality, let $u=x_{k-1}^{k+2}$. Consider the following paths:

(2.1) $w \sim x \sim y$

(2.2) $w \sim x_{i}^{k+1} \sim w_{i}^{k+2} \sim x_{i}^{k+2} \sim y$, for all $i \in\{1, \ldots, k-2\}$

(2.3) $w \sim x_{k-1}^{k+1} \sim w_{k-1}^{k+2} \sim u$

(2.4) $v \sim w_{k}^{k+2} \sim x_{k}^{k+2} \sim y$

(2.5) $v \sim w_{k}^{j} \sim x_{k}^{j} \sim w_{k+1}^{j} \sim x_{k-1}^{j} \sim w_{k-1, k+1}^{k+2, j} \sim u$, for all $j \in\{k+3, \ldots, 2 k+1\}$.

In each of the above two cases, it can be readily checked that there are $2 k$ paths and they are all internally disjoint. Thus, $|S| \geq 2 k$.

Lemma 4.2. Let $S$ be a minimum super vertex-cut of $\mathcal{G}=H(2 k+1, k)$, where $k \geq 2$. If $S$ contains a vertex $x$ such that $x$ has a neighbour in every component of $\mathcal{G}-S$ and $x \in V_{2}$, then $|S| \geq 2 k$.

Proof. Since $x \in V_{2}$, then $|x|=k+1$, say $x=z_{1} \ldots z_{k+1}$. Let a neighbour of $x$ in a component $C_{1}$ be $w$ and let a neighbour of $x$ in a component $C_{2}$ be $y$. Using a similar reasoning as in Lemma 4.1, without loss of generality, we can let $w=z_{1} \ldots z_{k}$ and $y=z_{1} \ldots z_{k-1} z_{k+1}=w_{k}^{k+1}$. Also, we let a vertex $v$ be adjacent to $w$ in $C_{1}$ such that $v \in V_{2}$ and, similarly, a vertex $u$ be adjacent to $y$ in $C_{2}$ such that $u \in V_{2}$, noting that $w \subset v$ and $y \subset u$. Thus, without loss of generality, we let $v=z_{1} \ldots z_{k} z_{k+2}$, leaving us with two separate cases to consider, according to whether $|u \cap v|=k$ or $|u \cap v|=k-1$.

Case 1. $|u \cap v|=k$, in which case $u=x_{k}^{k+2}$. Consider the following paths:

(1.1) $w \sim x \sim y$

(1.2) $w \sim x_{k+1}^{i} \sim w_{k}^{i} \sim x_{k}^{i} \sim y$, for all $i \in\{k+3, \ldots, 2 k+1\}$

(1.3) $v \sim w_{k}^{k+2} \sim u$

(1.4) $v \sim w_{j}^{k+2} \sim x_{j}^{k+2} \sim w_{j, k}^{k+1, k+2} \sim u$, for all $j \in\{1, \ldots, k-1\}$.

Case 2. $|u \cap v|=k-1$, in which case $u=x_{k}^{k+3}$. Consider the following paths:

(2.1) $w \sim x \sim y$

(2.2) $w \sim x_{k+1}^{k+3} \sim w_{k}^{k+3} \sim u$

(2.3) $w \sim x_{k+1}^{i} \sim w_{k}^{i} \sim x_{k}^{i} \sim y$, for all $i \in\{k+4, \ldots, 2 k+1\}$

(2.4) $v \sim w_{k}^{k+2} \sim x_{k}^{k+2} \sim y$

(2.5) $v \sim w_{j}^{k+2} \sim x_{j}^{k+2} \sim w_{j}^{k+1} \sim x_{j}^{k+3} \sim w_{j, k}^{k+1, k+3} \sim u$, for all $j \in\{1, \ldots, k-1\}$.

In each of the above two cases, it can be readily checked that there are $2 k$ paths and they are all internally disjoint. Thus, $|S| \geq 2 k$.

Theorem 4.3. The super-connectivity of the bipartite Kneser graph $H(2 k+1, k)$ is $2 k$, where $k \geq 2$.

Proof. The lower bound follows by Remark 2.1 together with Lemmas 4.1 and 4.2.

To see that the super-connectivity is at most $2 k$, it is sufficient to take the neighbours of two adjacent vertices to make up a super vertex-cut $S$. First, we note that $H(2 k+1, k)$ is regular of degree $k+1$ and that no two adjacent vertices share a common neighbour, implying that $|S|=2 k$. Also, $S$ cannot contain all the $k+1$ neighbours of any vertex in $H(2 k+1, k)-S$, because $H(2 k+1, k)$ is a bipartite graph and $S$ contains $k$ vertices from each of the two partite sets. Thus, no isolated vertices are created upon deleting $S$, and result follows.

\section{Conclusion}

From Theorems 2.2 and 2.3, it follows that the connectivity of the odd graph $O_{k+1}$ and of its Kronecker double cover $H(2 k+1, k)$ is equal to $k+1$. In Theorems 3.1 and 4.3 we have shown that their super-connectivity is equal to $2 k$. Thus, both the family of odd graphs and the family of the Kronecker double cover of odd graphs are super-connected. 


\section{REFERENCES}

[1] C. Balbuena and X. Marcote, The p-restricted edge-connectivity of Kneser graphs. Appl. Math. Comput. 343 (2019) $258-267$.

[2] F.T. Boesch, Synthesis of reliable networks - a survey. IEEE Trans. Reliab. 35 (1986) 240-246.

[3] F. Boesch and R. Tindell, Circulants and their connectivities. J. Graph Theory 8 (1984) 487-499.

[4] G. Boruzanlı Ekinci and J.B. Gauci, On the reliability of generalized Petersen graphs. Discrete Appl. Math. 252 (2019) $2-9$.

[5] G. Boruzanlı Ekinci and J.B. Gauci, The super-connectivity of Johnson graphs. Discrete Math. Theor. Comput. Sci. 22 (2020) 12.

[6] G. Boruzanlı Ekinci and J.B. Gauci, The super-connectivity of Kneser graphs. Discuss. Math. Graph Theory 39 (2019) 5-11.

[7] G. Boruzanlı Ekinci and A. Kırlangiç, Super connectivity of Kronecker product of complete bipartite graphs and complete graphs. Discrete Math. 339 (2016) 1950-195.

[8] X.L. Cao and E. Vumar, Super edge connectivity of Kronecker products of graphs. Int. J. Found. Comput. Sci. 25 (2014) $59-65$.

[9] B.L. Chen and K.-W. Lih, Hamiltonian uniform subset graphs. J. Comb. Theory, Ser. B. 42 (1987) $257-263$.

[10] C. Dalfó Simó, M.À. Fiol Mora and M.M. Riera, On middle cube graphs. Electron. J. Graph Theory App. 3 (2015) $133-145$.

[11] G. Gévay and T. Pisanski, Kronecker covers, V-construction, unit-distance graphs and isometric point-circle configurations. Ars Math. Contemp. 7 (2013).

[12] L. Guo and X. Guo, Super connectivity of Kronecker products of some graphs. Ars Comb. 123 (2015) 65-73.

[13] L. Guo, G. Su, W. Lin and J. Chen, Fault tolerance of locally twisted cubes. Appl. Math. Comput. 334 (2018) $401-406$.

[14] F. Harary, Conditional connectivity. Networks 13 (1983) 347-357.

[15] M.-C. Heydemann, Cayley graphs and interconnection networks, edited by G. Hahn and G. Sabidussi. In: Graph Symmetry: Algebraic Methods and Applications. Springer Netherlands, Dordrecht (1997) 167-224.

[16] J.-S. Kim, E. Cheng, L. Liptak and H.-O. Lee, Embedding hypercubes, rings, and odd graphs into hyper-stars. Int. J. Comput. Math. 86 (2009) 771-778.

[17] A. Kowalewski, WR Hamilton's Dodekaederaufgabe als Buntordnungproblem. Sitzungsber. Akad. Wiss. Wien (Abt. IIa) 126 (1917) 67-90, 963-1007.

[18] L. Lovász, Kneser's conjecture, chromatic number, and homotopy. J. Comb. Theory Ser. A. 25 (1978) $319-324$.

[19] M. Lü, C. Wu, G.-L. Chen and C. Lv, On super connectivity of Cartesian product graphs. Networks 52 (2008) $78-87$.

[20] S.M. Mirafzal, On the automorphism groups of regular hyperstars and folded hyperstars. Ars Comb. 123 (2011) 75-86.

[21] S.M. Mirafzal and A. Zafari, Some algebraic properties of bipartite Kneser graphs. Preprint arXiv: 1804.04570, to appear in Ars Combinatoria (2018).

[22] T. Mütze and P. Su, Bipartite Kneser graphs are Hamiltonian. Electron. Notes Discrete Math. 49 (2015) $259-267$.

[23] M.E. Watkins, Connectivity of transitive graphs. J. Comb. Theory 8 (1970) 23-29.

[24] W. Yang and J. Meng, Extraconnectivity of hypercubes. Appl. Math. Lett. 22 (2009) 887-891.

[25] W. Yang and J. Meng, Extraconnectivity of hypercubes (II). Australas. J. Comb. 47 (2010) 189-195. 